Theoretical Informatics and Applications

Theoret. Informatics Appl. 35 (2001) 379-388

\title{
ON THE EXPRESSIVE POWER OF THE SHUFFLE OPERATOR MATCHED WITH INTERSECTION BY REGULAR SETS
}

\author{
JoAnnA JȩDRZEJOWICZ ${ }^{1}$ AND ANDRZEJ Szepietowski ${ }^{1}$
}

\begin{abstract}
We investigate the complexity of languages described by some expressions containing shuffle operator and intersection. We show that deciding whether the shuffle of two words has a nonempty intersection with a regular set (or fulfills some regular pattern) is NL-complete. Furthermore we show that the class of languages of the form $L \cap R$, with a shuffle language $L$ and a regular language $R$, contains nonsemilinear languages and does not form a family of mildly contextsensitive languages.
\end{abstract}

Mathematics Subject Classification. 68Q15, 68Q45.

\section{INTRODUCTION}

Parallel composition of words appears to be an important issue both in the theory of concurrency and formal languages. Usually it is modeled by the shuffle operation. If the class of regular languages is extended by the shuffle operation $\odot$ and the iteration of the shuffle operation, $\otimes$, then we obtain the class of shuffle languages $S L$, which is useful in describing concurrent non-communicating processes [6]. We know [3] that for each shuffle language $L$ there exists a one-way nondeterministic Turing machine which decides the membership problem for $L$ in logarithmic space. This implies that shuffle languages are context sensitive and that they are recognizable in polynomial time. But the shuffle operation can be helpful to describe more complex languages. Warmuth and Haussler [8] show that if we add the intersection operation then we can obtain an NP-complete language, namely the language $\left\{\$ a^{n} b^{n} c^{n} d^{n} \mid n \geq 0\right\}^{\otimes}=\left((\$+a b c d)^{\otimes} \cap\left(\$ a^{*} b^{*} c^{*} d^{*}\right)\right)^{\otimes}$. They

Keywords and phrases: Formal languages, shuffle, space complexity.

${ }^{1}$ Institute of Mathematics, University of Gdańsk, ul Wita Stwosza 57, 80952 Gdańsk, Poland; e-mail: jj@math.univ.gda.pl \& matszp@math.univ.gda.pl 
also show that the problem of deciding for any words $w, w_{1}, \ldots, w_{n} \in\{a, b, c\}^{*}$ whether $w \in w_{1} \odot \cdots \odot w_{n}$ is NP-complete.

In this paper we consider some languages described with the help of both the shuffle operations and the intersection with regular sets. Firstly, we consider languages of the form $\{u \$ v \mid u \odot v \cap R \neq \emptyset\}$ or $\{u \$ v \mid u \odot v \subset R\}$, where $R$ is a regular language. The latter is the problem of deciding whether the shuffle of two words fulfills the regular pattern $R$. We show that every such language belongs to $N L$ (is acceptable in nondeterministic logarithmic space) and that there exists an $N L$-complete language of each of these forms. We also show that there are $N L$-complete languages of the form $\{u \mid u \odot u \cap R \neq \emptyset\}$ or $\{u \mid u \odot u \subset R\}$.

Furthermore we consider the class $S L \wedge \operatorname{Reg}=\{L \cap R \mid L \in S L, R \in R e g\}$ of languages represented as the intersection of a shuffle language and a regular one. The reason for starting this investigation was, as it seemed, the connection of $S L \wedge$ Reg with mildly context-sensitive languages considered in [4] and defined as follows: a family $\mathcal{L}$ is a mildly context-sensitive family of languages if each language in $\mathcal{L}$ is semilinear, and recognizable in deterministic polynomial time, and if $\mathcal{L}$ contains the following three languages: $L_{1}=\left\{a^{i} b^{i} c^{i} \mid i \geq 0\right\}$ (multiple agreements), $L_{2}=\left\{a^{i} b^{j} c^{i} d^{j} \mid i, j \geq 0\right\}$ (crossed agreements), and $L_{3}=\{w w \mid$ $\left.w \in\{a, b\}^{*}\right\}$ (duplications). The class $S L \wedge$ Reg seemed a good candidate for a family of mildly context-sensitive languages in view of fulfilling the condition of polynomial complexity of membership problem [3] and neat description of multiple agreements and crossed agreements by suitable expressions. In this paper we show that $S L \wedge R e g$ contains some non-semilinear languages and does not contain $L_{3}$. Hence $S L \wedge$ Reg does not form a family of mildly context-sensitive languages.

\section{Preliminaries}

Let $\Sigma$ be any fixed alphabet and $\lambda$ the empty word. By $u \cdot v$ we denote the concatenation of two words $u$ and $v$. We shall also use the notation $\prod_{i \in I} \sigma_{i}$, to denote the concatenation $\sigma_{i_{1}} \sigma_{i_{2}} \cdots \sigma_{i_{s}}$, where $I=\left\{i_{1}, i_{2} \ldots i_{s}\right\}$ and $i_{k}<i_{k+1}$ for every $1 \leq k \leq s-1$.

The shuffle operation $\odot$ is defined inductively as follows:

- $u \odot \lambda=\lambda \odot u=\{u\}$, for $u \in \Sigma^{*}$;

- $a u \odot b v=a(u \odot b v) \cup b(a u \odot v)$, for $u, v \in \Sigma^{*}$ and $a, b \in \Sigma$.

Note that $u \odot v$ consists of all words $z \in \Sigma^{*}$ which can be decomposed into $z=w_{1} \cdot w_{2} \cdots w_{r}$ with $w_{i} \in \Sigma^{*}, u=\prod_{i \in I} w_{i}$ and $v=\prod_{i \notin I} w_{i}$, for some subset $I \subset\{1,2, \ldots, r\}$. The shuffle operation is extended in a natural way to languages: for any languages $L_{1}, L_{2} \subset \Sigma^{*}$ the shuffle $L_{1} \odot L_{2}$ is defined as

$$
L_{1} \odot L_{2}=\bigcup_{u \in L_{1}, v \in L_{2}} u \odot v
$$


For any language $L$, the shuffle closure operator is defined by:

$$
L^{\otimes}=\bigcup_{i=0}^{\infty} L^{\odot i}, \quad \text { where } L^{\odot 0}=\{\lambda\} \text { and } L^{\odot i}=L^{\odot i-1} \odot L .
$$

Definition 1.1. Each $a \in \Sigma$, as well as $\lambda$ and $\emptyset$ are shuffle expressions. If $S_{1}, S_{2}$ are shuffle expressions, then $\left(S_{1} \cdot S_{2}\right), S_{1}{ }^{*},\left(S_{1}+S_{2}\right),\left(S_{1} \odot S_{2}\right)$ and $S_{1}{ }^{\otimes}$ are shuffle expressions, and nothing else is a shuffle expression.

The shuffle language $L(S)$ generated by a shuffle expression $S$ is defined as follows. $L(a)=\{a\}, L(\lambda)=\{\lambda\}, L(\emptyset)=\emptyset$. If $L\left(S_{1}\right)=L_{1}$ and $L\left(S_{2}\right)=L_{2}$, then $L\left(\left(S_{1} \cdot S_{2}\right)\right)=L_{1} \cdot L_{2}, \quad L\left(\left(S_{1}+S_{2}\right)\right)=L_{1} \cup L_{2}, \quad L\left(S_{1}{ }^{*}\right)=L_{1}^{*}, \quad L\left(\left(S_{1} \odot S_{2}\right)\right)=$ $L_{1} \odot L_{2}$, and $L\left(S_{1}{ }^{\otimes}\right)=L_{1}^{\otimes}$.

In what follows we shall not distinguish between the shuffle expression and the language generated by this expression. Shuffle languages are denoted by $S L$ and regular languages by $R e g$.

Assume that $\Sigma=\left\{a_{1}, \ldots, a_{n}\right\}$. The Parikh mapping, denoted by $\Psi$, is $\Psi$ : $\Sigma^{*} \rightarrow \mathbb{N}^{n}:$

$$
\Psi(w)=\left(\#_{a_{1}} w, \ldots, \#_{a_{n}} w\right),
$$

where $\#_{x} w$ denotes the number of occurrences of the letter $x$ in the word $w$.

For a language $L \subset \Sigma^{*}$ its Parikh image is defined by

$$
\Psi(L)=\bigcup_{w \in L} \Psi(w) .
$$

A linear set is a set $A \subseteq \mathbb{N}^{n}$ such that $A=\left\{v_{0}+\sum_{i=1}^{m} x_{i} v_{i} \mid x_{i} \in \mathbb{N}\right\}$ for some $v_{0}, v_{1}, \ldots, v_{m} \in \mathbb{N}^{n}$. A semilinear set is a finite union of linear sets and a language $L$ is semilinear if $\Psi(L)$ is a semilinear set. Observe that for any sets $A, B \subseteq \Sigma^{*}$, we have

$$
\begin{gathered}
\Psi(A \odot B)=\Psi(A B), \\
\Psi\left(A^{\otimes}\right)=\Psi\left(A^{*}\right),
\end{gathered}
$$

and since regular languages are semilinear, shuffle languages are semilinear, as well.

Example 1.2. Consider the language generated by the intersection of two shuffle expressions $S_{1}, S_{2}$ and a regular expression $R$ :

$$
\begin{gathered}
S_{1}=\left(\left((a b)^{\otimes} \odot X\right) Y\right)^{*}, \\
R=\left(a^{*} X b^{*} Y\right)^{*},
\end{gathered}
$$




$$
S_{2}=a X\left(\left((b a)^{\otimes} \odot Y\right) a X\right)^{*} b^{*} Y
$$

over the alphabet $\{a, b, X, Y\}$. It is easy to see that $S_{1} \cap R$ contains words of the form

$$
w=a^{n_{1}} X b^{n_{1}} Y \cdots a^{n_{k}} X b^{n_{k}} Y .
$$

If $w \in S_{2} \cap R$, then

$$
w=a X b^{m_{1}} Y a^{m_{1}+1} X b^{m_{2}} Y a^{m_{2}+1} \cdots b^{m_{l}} Y a^{m_{l}+1} X b^{m_{l+1}} Y .
$$

And if $w \in S_{1} \cap R \cap S_{2}$ we have $k=l+1$ (due to the number $\#_{Y} w$ ), besides $n_{1}=1, m_{1}=n_{1}=1, n_{2}=m_{1}+1=2, m_{2}=n_{2}=2, \ldots, n_{k}=m_{k-1}+1=k$. Thus

$$
S_{1} \cap R \cap S_{2}=\left\{a X b Y a^{2} X b^{2} Y \cdots a^{k} X b^{k} Y \mid k \geq 0\right\}
$$

is a non-semilinear language.

By $N S P A C E(\log n)$ or $N L$ we shall denote the class of languages accepted by nondeterministic Turing machines within logarithmic space and by one-way$N S P A C E(\log n)$ the class of languages accepted by one-way-nondeterministic Turing machines within logarithmic space.

\section{Finding the REgular PATtern in the ShUfFle of WORDS}

We consider the problem of deciding whether the shuffle of two words fulfills some regular pattern or has a nonempty intersection with a regular set. In other words the languages of the form $\{u \$ v \mid u \odot v \subset R\}$, or $\{u \$ v \mid u \odot v \cap R \neq \emptyset\}$ where $R \in R e g$ and $\$$ is a special separating symbol.

Theorem 2.1. For any regular language $R$ the sets $\{u \$ v \mid u \odot v \subset R\}$ and $\{u \$ v \mid$ $u \odot v \cap R \neq \emptyset\}$ are accepted in nondeterministic logarithmic space and there exists a NL-complete language of each of the above forms.

Proof. First we show that the language $\{u \$ v \mid u \odot v \cap R \neq \emptyset\}$ is accepted by a nondeterministic Turing machine $M$ in logarithmic space. The machine $M$ guesses one by one letters of a word $r$ of length $n=|u|+|v|$ and checks if $r$ belongs to $u \odot v \cap R$. To check if $r \in u \odot v, M$ uses two pointers; the first points to the successive letters of $u$, the second to the letters of $v$, at the beginning they point to the first letters of $u$ and $v$. $M$ guesses decomposition of $r=r_{1} \cdot r_{2} \cdots r_{n}$ into $\prod_{i \in I} r_{i}=u$ and $\prod_{i \notin I} r_{i}=v$, for some subset $I \subset\{1,2, \ldots, n\}$ and $r_{i} \in \Sigma$. In the $i$-th step $M$ guesses $r_{i}$ and whether $i \in I$ or not. If $i \in I$ then checks if the first pointer points to $r_{i}$ and moves this pointer right to the next position. If $i \notin I$, checks and moves the second pointer. In order to check if $r \in R, M$ simultaneously runs the finite automaton accepting $R$ on the successive letters of $r$. 
Note that

$$
\{u \$ v \mid u \odot v \subset R\}=\left\{u \$ v \mid u \odot v \cap R^{c} \neq \emptyset\right\}^{c} \cap\left(\Sigma^{*} \$ \Sigma^{*}\right) .
$$

Where $A^{c}$ is the complement of the language $A$. Using the fact that the class $N L$ is closed under complement and intersection with regular languages (see $[5,7])$ we have that the language

$$
\{u \$ v \mid u \odot v \subset R\}
$$

belongs to $N L$, as well.

Now we show that there is an $N L$-complete language of the form

$$
\{u \$ v \mid u \odot v \cap R \neq \emptyset\} .
$$

To do this we reduce the language GAP to the language

$$
L=\{u \$ v \mid u \odot v \cap R \neq \emptyset\}
$$

with the regular language

$R=d 1 d\left((a+b+e+D+E+0+1)^{*} e E(0 a+1 b)^{*} e E(d+e+0+1)^{*} d D(0 a+1 b)^{*} d D\right)^{*}$ over the alphabet $\{0,1, a, b, d, e, D, E\}$.

The language GAP consists of directed graphs $G=(V, E)$, which have a path from the first vertex to the last one. We shall assume that $V=\{1, \ldots, n\}$ for some $n$ and that if $(i, j) \in E$ then $i<j$ (even in this case GAP remains NL-complete, see [5]). The graph $G$ is coded in the following way

$$
G=d c(1) \operatorname{dec}\left(i_{1}^{1}\right) e \cdots e c\left(i_{j_{1}}^{1}\right) e \cdots d c(k) \operatorname{dec}\left(i_{1}^{k}\right) e \cdots e c\left(i_{j_{k}}^{k}\right) e \cdots d c(n) d,
$$

where $c(i)$ is the number $i$ coded in binary (note that $c(1)=1$ ), the symbols $d$ and $e$ play the role of the separators, and $i_{1}^{k}, \ldots, i_{j_{k}}^{k}$ are the vertices which are joined by an edge from $k$.

Reduction from GAP to $L=\{u \$ v \mid u \odot v \cap R \neq \emptyset\}$ is performed by the function $h(G)=u \$ v$ with $u=G$ and

$$
v=E C(1) E D C(1) D E C(2) E D C(2) D \cdots E C(n) E D C(n) D,
$$

where $C(i)$ is the number $i$ coded in binary with $a$ standing for 0 and $b$ standing for 1 and the symbols $D, E$ play the role of the separators,

First we show that if $G \in \mathrm{GAP}$ then there exists $r \in u \odot v \cap R$. Suppose that there is a path $1=s_{1}, s_{2}, \ldots, s_{t}=n$ joining 1 with $n$. Then there is a word

$$
r=U_{1} U_{2} \cdots U_{t} \in u \odot v \cap R
$$

where $U_{1}=d c(1) d$ and for each $i, 2 \leq i \leq t$

$U_{i} \in(a+b+e+D+E+0+1)^{*} e E(0 a+1 b)^{*} e E(d+e+0+1)^{*} d D(0 a+1 b)^{*} d D$ 
is of the form:

$$
U_{i}=v_{i} e E w_{i} e E x_{i} d D y_{i} d D,
$$

where:

- the part $e E w_{i} e E \in e E(0 a+1 b)^{*} e E$ is composed from $e c\left(s_{i}\right) e$ and $E C\left(s_{i}\right) E$, the part ec( $\left(s_{i}\right) e$ is taken from the list

$$
e c\left(i_{1}^{s_{i-1}}\right) e \ldots e c\left(i_{j_{s_{i-1}}}^{s_{i-1}}\right) e
$$

and $E C\left(s_{i}\right) E$ is taken from $v$;

- the part $d D y_{i} d D \in d D(0 a+1 b)^{*} d D$ comes from a shuffle of $d c\left(s_{i}\right) d$ (taken from $u$ ) and $D C\left(s_{i}\right) D$ (taken from $v$ );

- $x_{i} \in(d+e+0+1)^{*}$ is the fragment of $u$ standing between $e c\left(s_{i}\right) e$ and $d c\left(s_{i}\right) d$;

- $v_{i} \in(a+b+e+D+E+0+1)^{*}$ is the concatenation (or any other shuffle) of the fragment of $u$ standing between $d c\left(s_{i-1}\right) d$ and $e c\left(s_{i}\right) e$ and the fragment of $v$ standing between $D C\left(s_{i-1}\right) D$ and $E C\left(s_{i}\right) E$.

For every $i, 2 \leq i \leq t$ the pair $E C\left(s_{i}\right) E D C\left(s_{i}\right) D$ joins the element $e c\left(s_{i}\right) e$ from the list

$$
e c\left(i_{1}^{s_{i-1}}\right) e \cdots e c\left(i_{j_{s_{i-1}}}^{s_{i-1}}\right) e
$$

(remember that there is an edge from $s_{i-1}$ to $s_{i}$ and $e c\left(s_{i}\right) e$ is on the list) with the element $d c\left(s_{i}\right) d$ which stands in front of the list of vertices joined by an edge from $s_{i}$.

Now we show that if there exists $r \in u \odot v \cap R$ then there is a path from 1 to $n$ in $G$.

Since $r \in R$ then $r$ is of the form

$$
r=U_{1} U_{2} \cdots U_{t}
$$

with $U_{1}=d 1 d=d c(1) d$ and for each $i, 2 \leq i \leq t$

$U_{i} \in(a+b+e+D+E+0+1)^{*} e E(0 a+1 b)^{*} e E(d+e+0+1)^{*} d D(0 a+1 b)^{*} d D$

is of the form

$$
U_{i}=v_{i} e E w_{i} e E x_{i} d D y_{i} d D
$$

with $v_{i} \in(a+b+e+D+E+0+1)^{*}, w_{i} \in(0 a+1 b)^{*}, x_{i} \in(d+e+0+1)^{*}$, and $y_{i} \in(0 a+1 b)^{*}$.

Note that $e E w_{i} e E \in e E(0 a+1 b)^{*} e E$ can be only composed from $e c\left(\alpha_{i}\right) e$ and $E C\left(\gamma_{i}\right) E$ with ec $\left(\alpha_{i}\right) e$ taken from some list

$$
e c\left(i_{1}^{\beta_{i}}\right) e \ldots e c\left(i_{j_{\beta_{i}}}^{\beta_{i}}\right) e
$$

and $E C\left(\gamma_{i}\right) E$ taken from $v$. This composition is possible only if $\alpha_{i}=\gamma_{i}$ (ensured by $\left.w_{i} \in(0 a+1 b)^{*}\right)$. 
Similarly $d D y_{i} d D \in d D(0 a+1 b)^{*} d D$ can be only composed from $d c\left(\delta_{i}\right) d$ taken from $u$ and $D C\left(\epsilon_{i}\right) D$ taken from $v$. Note that this composition is possible only if $\delta_{i}=\epsilon_{i}$.

Observe that:

1. in $U_{2}$ there is no symbol $d$ in $v_{2}$, hence $e c\left(\alpha_{2}\right) e$ is taken from the first list in $u, \beta_{2}=1$, and there is an edge from 1 to $\alpha_{2}$;

2. in $U_{i}$ there is no symbol $E$ or $D$ in $x_{i}$, hence $\gamma_{i}=\epsilon_{i}$, and $\alpha_{i}=\delta_{i}$;

3. there is no symbol $d$ standing between last $D$ in $U_{i}$ and the first $e$ in $U_{i+1}$, hence $\beta_{i+1}=\delta_{i}=\alpha_{i}$, and $\alpha_{i+1}$ is taken from the list

$$
e c\left(i_{1}^{\alpha_{i}}\right) e \ldots e c\left(i_{j_{\alpha_{i}}}^{\alpha_{i}}\right) e
$$

so there is an edge between $\alpha_{i}$ and $\alpha_{i+1}$;

4. there is no symbol $d$ behind $d D y_{t} d D$ in $U_{t}$, hence $\delta_{t}=\alpha_{t}=n$.

Hence, we have shown that the sequence $1=\alpha_{1}, \alpha_{2}, \ldots, \alpha_{t}=n$ forms a path from 1 to $n$ and that the language $L$ is NL-complete.

Because $N L$ is closed under complement the language non-GAP is also NLcomplete. It is easy to see that the function $h$ reduces non-GAP into

$$
\left\{u \$ v \mid u \odot v \subset R^{c}\right\}
$$

and thus this language is also NL-complete.

Consider now the languages of the form

$$
\{u \mid u \odot u \cap R \neq \emptyset\}
$$

with some regular language $R$. Of course every such language belongs to $N L$ and also among such languages there are $N L$-complete ones. Let

$$
T=X(0+1+d+e)^{*} Y X R Z Y(a+b+D+E)^{*} Z
$$

where $X, Y, Z$ are new separating symbols. It is easy to see that if $w=X u Y v Z$ then $w \odot w \cap T \neq \emptyset$ if and only if $u \odot v \cap R \neq \emptyset$. Hence we have:

Corollary 2.2. For any regular language $R$ the sets $\{u \mid u \odot u \subset R\}$ and $\{u \mid$ $u \odot u \cap R \neq \emptyset\}$ are accepted in nondeterministic logarithmic space and there exists a NL-complete language of each of the above forms.

\section{INTERSECTIONS WITH REGULAR LANGUAGES}

Now we consider the class $S L \wedge \operatorname{Reg}=\{L \cap R \mid L \in S L, R \in R e g\}$ of languages represented as the intersection of a shuffle language and a regular one. The reason 
for starting this investigation was, as it seemed, the connection of $S L \wedge R e g$ with mildly context-sensitive languages considered in [4] and defined as follows:

Definition 3.1. A family $\mathcal{L}$ is a mildly context-sensitive family of languages if the following conditions are fulfilled:

- each language in $\mathcal{L}$ is semilinear;

- for each language in $\mathcal{L}$ the membership problem is solvable in deterministic polynomial time, and

- $\mathcal{L}$ contains the following three non-context-free languages

- $L_{1}=\left\{a^{i} b^{i} c^{i} \mid i \geq 0\right\}$ (multiple agreements);

$-L_{2}=\left\{a^{i} b^{j} c^{i} d^{j} \mid i, j \geq 0\right\}$ (crossed agreements);

- $L_{3}=\left\{w w \mid w \in\{a, b\}^{*}\right\}$ (duplications).

The class $S L \wedge R e g$ seemed a good candidate for a family of mildly context-sensitive languages in view of fulfilling the condition of polynomial complexity of membership problem and neat description of multiple agreements and crossed agreements by suitable expressions. By [3], SL $\subset$ one-way- $N S P A C E(\log n)$, and the class one-way- $N S P A C E(\log n)$ is closed under intersections with regular languages [7], so $S L \wedge R e g \subset$ one-way- $N S P A C E(\log n) \subset P$.

The languages: multiple agreements $L_{1}$ and crossed agreements $L_{2}$ are in $S L \wedge$ Reg since

$$
\begin{gathered}
L_{1}=(a b c)^{\otimes} \cap a^{*} b^{*} c^{*}, \\
L_{2}=(a c+b d)^{\otimes} \cap a^{*} b^{*} c^{*} d^{*} .
\end{gathered}
$$

But the language $L_{3}$ does not belong to one-way-NSPACE $(\log n)$ [7], hence $L_{3}$ is not in $S L \wedge R e g$. It is interesting that $S L \wedge R e g$ contains two languages which are similar to duplicates.

Suppose that for any letter $a \in \Sigma$ we introduce a new symbol $a^{\prime}$ and denote $\Sigma^{\prime}=\left\{a^{\prime} \mid a \in \Sigma\right\}$. By a primed word we understand a word where all the symbols of the original word are replaced by primed symbols, that is if $w=a_{1} \cdots a_{n} \in \Sigma^{*}$, then $w^{\prime}=a_{1}^{\prime} \cdots a_{n}^{\prime} \in \Sigma^{\prime *}$.

Example 3.2. The following language $L \subseteq\left(\Sigma \cup \Sigma^{\prime}\right)^{*}$, of non-duplicates, is in the class $S L \wedge$ Reg:

$$
\left\{u v^{\prime} \mid v^{R} \neq u\right\}=\left(\bigcup_{a, b \in \Sigma, a \neq b} \Sigma^{*} a X^{\otimes} b^{\prime} \Sigma^{\prime *}\right) \cap \Sigma^{*} \Sigma^{\prime *},
$$

where $X=\left\{x x^{\prime} \mid x \in \Sigma\right\}$. Also the language of 'almost' duplicates is in $S L \wedge R e g$ :

$$
\left\{w v^{\prime} \mid v \in \operatorname{com}(w)\right\}=E Q(\Sigma) \cap \Sigma^{*} \Sigma^{\prime *},
$$

where $\operatorname{com}(w)=\left\{u \in \Sigma^{*} \mid\right.$ for each $\left.a \in \Sigma, \#_{a} w=\#_{a} u\right\}$ is the commutative closure of $w$ and $E Q(\Sigma)=\left(a_{1} a_{1}^{\prime}+\cdots+a_{n} a_{n}^{\prime}\right)^{\otimes}$, for $\Sigma=\left\{a_{1}, \ldots, a_{n}\right\}$. 
Now we show that $S L \wedge \operatorname{Reg}$ contains non-semilinear languages. Araki and Tokura proved the following:

Theorem 3.3. [1] For any alphabet $\Sigma$ and each recursively enumerable language $K$ over $\Sigma$ there exist two alphabets $\Gamma=\left\{\left[{ }_{1},[2, \ldots,[c, 1], 2], \ldots, c\right]\right\}, \Delta=$ $\left\{\sigma_{1}, \omega_{1}, \ldots, \sigma_{d}, \omega_{d}\right\}$ and a shuffle expression $S$ over $\Sigma \cup \Gamma \cup \Delta$ such that

$$
\begin{aligned}
& K=\left\{x_{1} \cdots x_{k} \in \Sigma^{*} \mid \text { there exist } y_{1}, \ldots, y_{k} \in(\Gamma \cup \Delta)^{*},\right. \\
& \text { such that } \left.x_{1} y_{1} \cdots x_{k} y_{k} \in S, \text { and } y_{1} \cdots y_{k} \in S_{\text {lock }} \odot S_{\text {signal }}\right\},
\end{aligned}
$$

where $S_{\text {lock }}=\left(\left[_{1_{1}}\right]\right)^{*} \odot \cdots \odot\left(\left[_{c c}\right]\right)^{*}, S_{\text {signal }}=\left(\sigma_{1}+\sigma_{1} \omega_{1}\right)^{*} \odot \cdots \odot\left(\sigma_{d}+\sigma_{d} \omega_{d}\right)^{*}$.

Note that $K$ can be expressed in the form

$$
K=\operatorname{er}_{\Sigma}(S \cap R),
$$

where $e r_{\Sigma}$ is a weak identity or erasing homomorphism defined by

$$
\operatorname{er}_{\Sigma}(x)= \begin{cases}x & \text { if } x \in \Sigma \\ \lambda & \text { otherwise }\end{cases}
$$

and $R=S_{\text {lock }} \odot S_{\text {signal }} \odot \Sigma^{*}$ is regular, because the shuffle of regular languages is regular.

Take now any non-semilinear recursively enumerable language $K$, by the above theorem it can be represented by

$$
K=e r_{\Sigma}(L)
$$

for some $L \in S L \wedge R e g$. Since the erasing homomorphism preserves semilinearity the language $L$ is also non-semilinear. Hence we have:

Corollary 3.4. There exists a non-semilinear language in the class $S L \wedge$ Reg.

Corollary 3.5. The class $S L \wedge$ Reg does not form a family of mildly contextsensitive languages.

Remark. We think that Theorem 3.3 can be strengthened in the following way. For any alphabet $\Sigma$ there exists an alphabet $\Gamma \supset \Sigma$ and a shuffle language $S$ over $\Gamma$ which is a "very special language" ([2]), that is for each recursively enumerable language $K$ over $\Sigma$ there exists a regular language over $\Gamma$ such that $K=\operatorname{er}_{\Sigma}(S \cap R)$.

\section{Open PROBLEMS}

1. Is there an NL-complete language in the class SL?

By [3] $S L \subset N L$. This problem is equivalent to the problem whether there is an NL-complete language in $S L \wedge R e g$. Indeed suppose that there exists a reduction $h$ from GAP to $L \cap R \subset \Sigma^{*}$ with some $L \in S L$ and $R \in R e g$, and let $\$$ be an arbitrary symbol not in $\Sigma$. Now, there exists the reduction $g$ from GAP to the shuffle 
language $L \subset(\Sigma \cup\{\$\})^{*}$ : on the input $x, g$ computes $h(x)$, checks simultaneously if it belongs to $R$, and sets $g(x)=h(x)$ if $h(x) \in R$, and $g(x)=h(x) \$$ otherwise.

2. Is there an NL-complete language of the form

$$
L=\left\{u \$ v_{1} \$ \cdots \$ v_{k} \mid u \in v_{1} \odot \cdots \odot v_{k}, \text { and } u, v_{1}, \ldots v_{k} \in \Sigma^{*}\right\}
$$

for some constant $k$ and some alphabet $\Sigma$ ?

Similarly as in the proof of Theorem 2.1 one can show that $L \in N L$. By [8], $L$ is $N P$-complete, if $k$ is not a constant and $\Sigma=\{a, b, c\}$.

\section{REFERENCES}

[1] T. Araki and N. Tokura, Flow languages equal recursively enumerable languages. Acta Inform. 15 (1981) 209-217.

[2] D. Haussler and P. Zeiger, Very special languages and representations of recursively enumerable languages via computation stories. Inform. and Control 47 (1980) 201-212.

[3] J. Jędrzejowicz and A. Szepietowski, Shuffle languages are in P. Theoret. Comput. Sci. 250 (2001) 31-53.

[4] C. Martin-Vide and A. Mateescu, Special families of sewing languages, in Workshop - Descriptional complexity of automata, grammars and related structures. Magdeburg (1999) 137-143.

[5] C.H. Papadimitriou, Computational Complexity. Addison-Wesley Publ. Co (1994).

[6] A.C. Shaw, Software descriptions with flow expressions. IEEE Trans. Software Engrg. 3 (1978) 242-254.

[7] K. Wagner and G. Wechsung, Computational Complexity. Reidel, Dordrecht, The Netherlands (1986).

[8] M.K. Warmuth and D. Haussler, On the complexity of iterated shuffle. J. Comput. Syst. Sci. 28 (1984) 345-358.

Communicated by Ch. Choffrut.

Received December, 2000. Accepted October, 2001. 